BULLETIN (New Series) OF THE

AMERICAN MATHEMATICAL SOCIETY

Volume 47, Number 1, January 2010, Pages 139-144

S 0273-0979(09)01286-5

Article electronically published on October 15, 2009

\title{
VON NEUMANN'S COMMENTS ABOUT EXISTENCE AND UNIQUENESS FOR THE INITIAL-BOUNDARY VALUE PROBLEM IN GAS DYNAMICS
}

\author{
DENIS SERRE
}

The context is that of a conference, Problems of Cosmical Aerodynamics, held in Paris, France, on August 16-19, 1949. It was organized at the initiative of the newly founded IUTAM 1 and of the IAU2. It gathered fifty-two scientists, among which there were thirty-four astronomers and eighteen physicists or fluid mechanicists. The chairs were J. M. Burgers for IUTAM and H. C. van de Hulst for IAU. Among the participants were W. Heisenberg, J. H. Oort, E. Schatzman, T. von Kármán, C. F. von Weizsä́cker and, of course, J. von Neumann. G. I. TAYLOR could not attend the conference because of sickness.

The conference was managed in a rather informal style, the program being decided during the first day, a little bit like our Oberwolfach workshops. Discussion sessions happened every day in the afternoon. I wish to comment here on the session chaired by VON NEUMANN, devoted to the Existence and uniqueness or multiplicity of solutions of the aerodynamical equations. The records of the discussion are reprinted in this issue after these comments. They are taken from pp. 75-85 of the conference proceedings, published by the Central Air Documents Office, Dayton (Ohio), 1951.

Instead of a discussion, the session was actually a long monologue by vON NEUMANN, after which he apologize(s) for having taken up so much of the limited time 3 He then answers the questions of a handful of scientists. The session ends with a comment by BuRgERs about the consequences of this discussion for astronomical problems.

In order to make the context as clear as possible, it is necessary to recall that during WWII, vON NEUMANN had been involved in the Manhattan Project, dedicated to the construction of the American nuclear bomb, while HeISENBERG led the German nuclear weapon program, to which WeIzsÄckER collaborated. This was the origin of their common, though independent, interest in fluid mechanics and shock waves. However, the 1949 conference had a peaceful target, and the conversations remained courteous. In addition, Germany was no longer a threat, thus it became meaningful to involve German scientists in such a conference. The

Received by the editors September 8, 2009.

2010 Mathematics Subject Classification. Primary 01A60, 35L65, 35L67, 35Q35, 35Q85, 76N10, 76N15, 76P05, 85-03, 85A30.

${ }^{1}$ International Union of Theoretical and Applied Mechanics.

${ }^{2}$ International Astronomical Union.

${ }^{3}$ From now on, italics indicate quotations from the proceedings. 
situation was the opposite with the USSR, as the Cold War had begun immediately after victory. This must be the reason for the lack of Eastern scientists in this meeting, despite the quality of the Soviet research, especially in physics and astrophysics.

The discussion is about models for fluid mechanics, mostly the Euler system. Since the latter does not appear explicitly in the records, I recall it for the comfort of the readers. It governs the evolution of the mass density $\rho$, the fluid velocity $\vec{u}=\left(u_{1}, \ldots, u_{d}\right)$ and the specific internal energy $e$. These unknowns are functions of the time $t$ and the space variables $\left(x_{1}, \ldots, x_{d}\right)$. The space dimension $d$ equals 3 in practical applications. The equations express the conservation of mass, momentum and total energy:

$$
\begin{aligned}
\frac{\partial \rho}{\partial t}+\operatorname{div}_{x}(\rho \vec{u}) & =0, \\
\frac{\partial \rho u_{j}}{\partial t}+\operatorname{div}_{x}\left(\rho u_{j} \vec{u}\right)+\frac{\partial p}{\partial x_{j}} & =0, \quad \forall j=1, \ldots, d, \\
\frac{\partial}{\partial t}\left(\frac{1}{2} \rho|\vec{u}|^{2}+\rho e\right)+\operatorname{div}_{x}\left(\frac{1}{2} \rho|\vec{u}|^{2}+\rho e+p\right) \vec{u} & =0 .
\end{aligned}
$$

The auxiliary quantity $p$, the pressure of the fluid, is a given function of the internal variables $(\rho, e)$. With the chain rule, a combination of the equations above yields a transport equation

$$
\left(\frac{\partial}{\partial t}+\vec{u} \cdot \nabla_{x}\right) s=0
$$

where $s=s(\rho, e)$, the entropy of the fluid, is a state function obeying the differential equation

$$
\left(p \frac{\partial}{\partial e}+\rho^{2} \frac{\partial}{\partial \rho}\right) s=0
$$

Equation (4) suggests that if

$$
x \mapsto s(\rho(x, 0), e(x, 0))
$$

is a constant, then $s(\rho(x, t), e(x, t))$ is a constant too. This thought was known to be too naïve in 1949, as we shall see below. When $s$ is taken as a constant, then (3) is formally a consequence of (112); we speak of an "isentropic flow".

The equations (11) -(3) have the general form of "conservation laws" $\operatorname{div}_{(x, t)} \mathbf{Q}_{\alpha}=$ 0 for $\alpha=1, \ldots, d+2$. It is easy to consider discontinuous solutions, using the integral form (Green's formula). Discontinuities obey to jump conditions: the normal component of the vector field $\mathbf{Q}_{\alpha}$ is continuous. We remark that a combination of (11) and (4) yields another conservation law,

$$
\frac{\partial \rho s}{\partial t}+\operatorname{div}_{x}(\rho s \vec{u})=0 .
$$

The main concern of VON NEUMANN derives from the lack of existence and uniqueness theorems to either the Cauchy problem or the initial-boundary value problem (IVBP) for (11)-(3). They have never been demonstrated and are probably not true in their obvious form.

His second paragraph begins with an homage to B. RIEMANN, through a sentence that deserves an explanation: In this case [namely an isentropic flow in one space dimension] ... Riemann proved that there are no discontinuities. Twenty-first 
century scientists would disagree at first sight, since we know that for a differentiable initial data, a differentiable solution exists in a certain time interval, but that discontinuities develop in finite time, in general. What vON NEUMANN actually means is that RIEMANN proved that, although for a classical solution of the full Euler equations the entropy may be a constant, the latter cannot be so if this solution experiences a discontinuity. The reason is that the jump conditions for (11)-(3) across the discontinuity locus are not compatible if $s$ is constant. In other words, the jump conditions for (11)-(3) and (5) are not compatible.

Von Neumann recalls the lack of a solution in general. Again, this is a little bit difficult to understand for us, as we immediately think of "weak entropy solution" when hearing the word "solution". But vON NEumanN has in mind a global-intime classical solution. This becomes clear in the last two sentences. Because the audience consisted mostly of astronomers who were not acquainted with shock theory, he recalls the impossibility for the entropy to decay in a classical solution. This, together with the apparent reversibility of the system, would imply the constancy of the entropy along the particle path, raising ultimately an obstruction to the continuation of the classical solution. Whence the necessity of considering discontinuous ones.

Next, vON NEUMANN recalls the other astonishing facet of gas dynamics: if we permit discontinuities in an attempt to recover the global existence of solutions, then the nonexistence is turned into a nonuniqueness. This motivates the second principle of thermodynamics, according to which the entropy may not decrease along a particle path. Its role is to select the physically admissible discontinuities or, equivalently, to put a small amount of irreversibility in the Euler system. This is a beautiful example of how the reversible system of mechanics can yield a description of an irreversible piece of nature. The first works making this sentence a theory came a few years later, with the celebrated papers by P. LAX (1954) and S. KRUZKHOV (1970). With a certain amount of optimism, which is the mark of great minds, VON NEUMANN anticipates There probably exists a set of conditions under which one and only one solution exists [...] However [...], we have to be guided almost entirely by physical intuition in searching for it. The first part of this quotation is still the subject of intensive research, the case of one space variable being now well understood. The existence part was proved by J. GLimm (1965), precisely by a deep use of physical intuition 5 while the uniqueness and continuous dependence upon the data are due to A. BRESSAN and his coworkers (1995-2000). These great achievements leave however a bitter taste in the mouth, as they are limited to solutions and data of bounded variations in one space dimension. J. RAUCH (1986) showed that this functional framework is not adapted to the multidimensional context. In addition, existence is known only when the total variation of the initial data is "small". An alternate existence result, valid for arbitrarily large initial data, was obtained by R. DiPerna (1983), still in one dimension, with perhaps more mathematical insight, but the lack of regularity of such solutions has not permitted yet the establishment of a uniqueness theorem 6 in the same functional framework.

\footnotetext{
${ }^{4}$ Invariance under the transformation $(x, t, \rho, \vec{u}, e) \mapsto(x,-t, \rho,-\vec{u}, e)$.

${ }^{5}$ The so-called "potential of interaction", crucial in the a priori estimates, is primarily a physical concept.

${ }^{6}$ To paraphrase vON NEUMANN, such a uniqueness result is probably not true.
} 
In addition, it applies to isentropic flows only, which are unphysical in the presence of shock waves, as mentioned above.

Mathematically, the second principle of thermodynamics amounts to replacing (51) by an inequality in the sense of distributions:

$$
\frac{\partial \rho s}{\partial t}+\operatorname{div}_{x}(\rho s \vec{u}) \geq 0 .
$$

This is possible because, when dropping the differentiability, (5) is no longer a consequence 7 of (11)-(3). This formulation was unclear at the time of the conference 8 and VON NEUMANN said (on page 77 of the original), There is no mathematical reason at all why you should not use a solution of that type [with an expansive discontinuity], but it will lead to a decrease of entropy. Condition (6) is a case of the so-called "Lax entropy inequality", which occurs in the more general context of hyperbolic systems of conservation laws.

The next paragraph is a very interesting discussion about the relevance of shock waves, which are admissible discontinuities. First of all, vON NEUMANN recalls the works by RAYLEIGH and G. I. TAYLOR (1910) and others about their internal structure when viscosity and heat conductivity are taken in account 9 The existence of such a structure, now called a "shock profile", has become in the past few decades an efficient admissibility criterion; it is in general more restrictive than the Lax entropy inequality. At a smaller scale, the gas is even not at thermodynamical equilibrium, and VON NEUMANN observes accurately, in the first few mean free paths, [...] there is not a discontinuity at all, but a region where deviations from the classical Maxwell-Boltzmann velocity distribution of the kinetic theory of gases occur. The mathematical justification of this assertion is still incomplete.

The last question raised by vON NEUMANN concerns the validity of the Euler system in the presence of a vacuum (top of page 78). If we are in the expanding gas [advancing into a vacuum] and approach the (theoretical) front, we will necessarily come to regions where the mean free path is larger than the distance from the front. In such regions one cannot use the hydrodynamical equations. This statement is the matter of subsequent exchanges between VON NEUMANN and several participants, including HEISENBERG.

To finish his long introduction, vON NEUMANN mentions physical experiments which confirm the exact solutions that can be derived analytically from the equations: the Riemann solution of the piston problem and the Prandtl-Meyer expansion fan around a convex corner.

The discussion. The contributors have been H. W. Liepmann, G. Temple, von Kármán, Heisenberg, Burgers and, of course, von Neumann himself.

Personally, I like VON KÁRMÁN's assessment: I don't think that there is any reason that if you put a problem in a form which has no physical meaning, there shall not be two solutions. Somehow, it is a contraposition of E. Wigner's comment about the "unreasonable effectiveness of mathematics in the natural sciences". Applied mathematicians - including specialists of partial differential equations, of probability, or of many other fields - should keep it in mind. VON KÁRMÁN raises doubts about the relevance of some mathematical problems, like shock reflection

\footnotetext{
${ }^{7}$ Because the chain rule is not available.

${ }^{8}$ Before the theory of distributions was popularized by L. SCHWARTz.

${ }^{9}$ With this completion, the Euler system becomes the Navier-Stokes-Fourier system.
} 
against an infinite wall or against a corner bounded by semi-infinite walls. VON NEUmanN insists about the nonuniqueness in shock reflection problems. He presumably thought of the regular reflection, where algebraic calculations provide a weak reflection as well as a strong one; the former case only is observed in physical experiments, whereas this selection has never been explained at an analytical level. In the reviewer's opinion, this is not really a nonuniqueness phenomenon for an IBVP, since the strong reflection is only a local-in-space and time solution. In an answer to BURGERS' suggestion to consider the shock reflection against a corner, VON NEUmANn is more cautious: In that case you assume that the state at the time $t=0$ is given and you ask whether there is or is not a unique continuation of the solution at later times. The answer to this question in its full generality is not known; there seem to be great many mathematical difficulties. The countless works on this problem, while we still lack an answer, confirm VON NEumanN's impression. So far, the only existence result (G.-Q. Chen and M. Feldman, 2005) concerns the interesting but unphysical irrotational case.

Another important comment is made by HEISENBERG about the role played by the boundary conditions in the selection process. This is reminiscent of the boundary layer phenomenon, which is still poorly understood. vON NEUMANN answers: The boundary layer theory for a fluid of low viscosity certainly furnishes a monumental warning. The naive and yet prima facie seemingly reasonable procedure would be to apply the ordinary equations of the ideal fluid and then to expect that viscosity will somehow take care of itself in a narrow region along the wall. We have learned that this procedure may lead to great errors; a complete theory of the boundary layer may give you completely different conditions also for the flow in the bulk of the field. It is possible that the same discipline will be necessary for the boundary with a vacuum.

As a conclusion, BURGERS, who I recall was one of the organizers, observes: the discussion of this morning [...] has centered about the properties of certain hydroor aerodynamical equations and about certain solutions. It may be useful to ask ourselves what does this mean for astronomy.

Conclusion. Sixty years after, we read these records as if they had been written a decade ago. In a few pages, all the important questions are raised and examples are given, which still serve as paradigms. The main conclusion is still valid: what is important is the real world, that is physics, but it can be explained only in mathematical terms. We need a rigorous mathematical theory of aerodynamical flows, in agreement with physical experiments. While flows with a reasonable regularity are well described by continuum equations (Euler or Navier-Stokes-Fourier, depending on scale), we need to incorporate additional ingredients to render a correct account of shock waves, boundary layers and vaccum, at least. It is fascinating that these questions are still widely open, even if our understanding is much better now than 60 years ago.

\section{REFERENCES}

[1] A. Bressan. Hyperbolic systems of conservation laws. Oxford Lecture Series in Mathematics and its Applications, 20. Oxford University Press, Oxford (2000). MR.1816648(2002d:35002)

[2] G.-Q. Chen and M. Feldman. Potential theory for shock reflection by a large-angle wedge. Proc. Nat. Acad. Sci. USA, 102 (2005), pp. 15368-15372. MR2188921(2006f:35214) 
[3] R. J. DiPerna. Convergence of the viscosity method for isentropic gas dynamics. Comm. Math. Phys., 91 (1983) pp. 1-30. Convergence of approximate solutions to conservation laws. Arch. Rat. Mech. Anal., 82 (1983), pp. 27-70. MR.719807 (85i:35118)

[4] J. Glimm. Solutions in the large for nonlinear hyperbolic systems of equations. Comm. Pure Appl. Math., 18 (1965), pp. 697-715. MR0194770 (33:2976)

[5] S. Kružkov. Generalized solutions of the Cauchy problem in the large for nonlinear equations of first order. Dokl. Akad. Nauk SSSR, 187 (1969), pp. 29-32. MR0249805 (40:3046)

[6] P. D. Lax. Hyperbolic systems of conservation laws (II). Comm. Pure Appl. Math., 10 (1957), pp. 537-566. MR0093653 (20:176)

[7] J. Rauch. BV estimates fail for most quasilinear hyperbolic systems in dimensions greater than one. Comm. Math. Phys., 106 (1986), pp. 481-484. MR859822 (87m:35139)

École Normale Supérieure de Lyon, D. S.: UMPA, CNRS UMR 5669. ENS de Lyon, 46 allée d'Italie. F-69364 Lyon, Cedex 07, France 